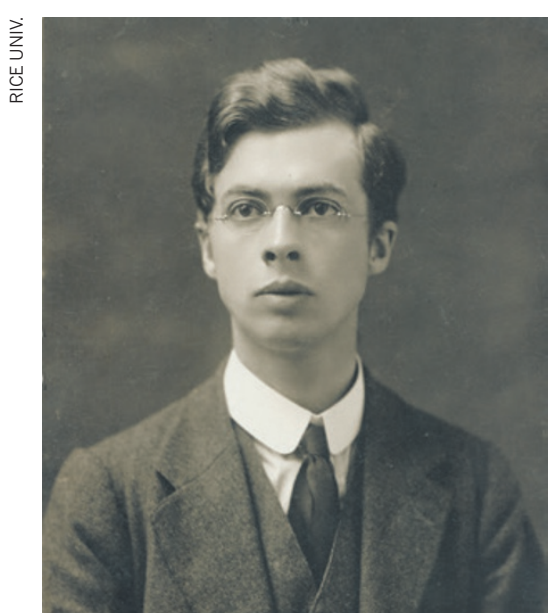

Julian Huxley in 1914.

published a paper in The American Naturalist that effectively poured cold water on any enthusiasm for female choice, and even more for mutual choice.

There matters largely rested until the early 1980s, when Malte Andersson showed that female choice did select for extreme male tail length in the African widowbird (Euplectes progne). Ten years later, Ian Jones and Fiona Hunter studied the crested auklet (Aethia cristatella), a monogamous seabird in which both sexes are ornamented. The parallels with the great crested grebe are obvious. Jones and Hunter showed that both males and females responded to accentuated models of the opposite sex with more frequent displays - confirming that ornaments in both sexes could be favoured by mutual mating preferences. Although beyond the scope of that study, it also seemed possible that ornament size influenced the likelihood of re-pairing the following year. That would have further undermined Huxley's reluctance to concede a role for sexual selection in any display after birds have paired for the season.

In 1946, Huxley became the first director of the fledgling United Nations Educational, Scientific and Cultural Organization (UNESCO), and he was instrumental in the 1961 founding of the World Wildlife Fund, now known as WWF. In the field of evolution, his overwhelming influence may have put a brake on the study of sexual selection, and his grebe-courtship observations have largely been superseded by those of zoologist Ken Simmons. But nobody has put two weeks' birdwatching in the spring sunshine beside an English reservoir to greater heuristic effect.

Michael Brooke is Strickland Curator of Ornithology at the University Museum of Zoology in Cambridge, UK.

e-mail:m.brooke@zoo.cam.ac.uk

\section{A few words on infinity}

\author{
Lucy Fortson enjoys a slim primer on cosmology that \\ uses a cleverly constrained lexicon.
}

$\mathrm{W}$

hen we think of cos-
mology, we might
think of some of its most complex scientific theories, such as inflation or the general theory of relativity. Or we might consider its astounding discoveries, such as the twinned mysteries of dark matter and dark energy, which together comprise $96 \%$ of the contents of the Universe. We may then have the unsettling idea that the remaining $4 \%$ (including the stuff of which humanity is made) is insignificant in the grand scheme of things - a thought all the more remarkable when we consider the extent to which the bits of recycled stars known as humans understand cosmology.

The extraordinary story of the Universe and our journey to understand it is not an easy one to tell to the general public. But because it is the story that binds us all together, it is important to tell it in myriad ways to reach as many people as possible. The Edge of the Sky is an inventive, enjoyable and thought-provoking contribution to that effort.

Inspired by 'Up Goer Five' - an instalment of the webcomic $x k c d$, by former NASA roboticist Randall Munroe - theoretical cosmologist Roberto Trotta uses fewer than 90 pages to take the reader through a strange, yet sometimes compelling, exercise. He translates our current understanding of cosmology into the 1,000 most popular words in English (or as the book would say, "the ten hundred most-used words in our tongue"). Effectively, this approach demanded the invention of a new language through renaming of common objects: aeroplanes, for instance, become flying cars. It is as if we are reading a book by an observer on a different planet. Paradoxically, this simplicity of language encourages us to think outside the familiar.

So we follow a "student-person" (scientist) through one

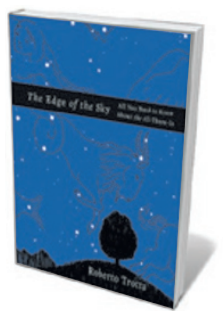

The Edge of the Sky: All You Need to Know about the All-There-Is Basic Books: 2014 ROBERTO TROTTA night of observation using a "BigSeer" (telescope), and her reflections on the series of "Why? questions" (science) that have led her, and humanity, to the point of understanding as much as we do about the "All-There-Is" (Universe). We move from early (Western) cosmology that the "old people" believed in, centred on "Crazy Stars" (planets), to the discoveries of planets outside the Solar System and today's search for new "Home-Worlds". We then follow a fairly conventional path with the usual suspects, including the "student-people" Mr Hubble, Mr Einstein and Mrs Rubin, as Trotta brings us through discoveries from the expansion of the Universe to Big Bang nucleosynthesis - all using a total of just 707 different words (and 42 names).

This linguistic constriction left me wondering about the intended readership. At times, the exercise feels like just that, yielding pained oversimplifications that give the impression of inaccuracy, such as "tired light" for redshift. We begin to wonder whether Trotta embarked on the project merely as an intellectual puzzle, proving to himself that he could write a satisfactory explanation of cosmology with an arbitrary constraint on vocabulary. (There are faint echoes here, for instance, of the French writer and filmmaker Georges Perec's experimental 1969 novel La Disparition, which excludes the letter e.)

In reading this book, do the cosmologically uninitiated really gain a clearer understanding of the workings of the Universe, and could they then explain this to someone else using ordinary terminology? Probably not. But, as with a well-told folk tale, perhaps some of the passion and poetry of this ultimate quest will be conveyed, inspiring a new student-person to ask the right Why? questions. This would be no small success.

Lucy Fortson is associate professor of physics at the University of Minnesota in Minneapolis, where she teaches cosmology. She is also committed to improving the scientific literacy of the general public through the citizen-science project Zooniverse (www.zooniverse.org). e-mail:fortson@physics.umn.edu 